\title{
Golog Speaks the BDI Language
}

\author{
Sebastian Sardina ${ }^{1}$ and Yves Lespérance ${ }^{2}$ \\ 1 Department of Computer Science and IT \\ RMIT University \\ Melbourne, Australia \\ sebastian.sardinalrmit.edu.au \\ 2 Department of Computer Science and Engineering \\ York University \\ Toronto, Canada \\ lesperan@cse.yorku.ca
}

\begin{abstract}
In this paper, we relate two of the most well developed approaches to agent-oriented programming, namely, BDI (Belief-Desire-Intention) style programming and "Golog-like" high-level programming. In particular, we show how "Golog-like" programming languages can be used to develop BDI-style agent systems. The contribution of this paper is twofold. First, it demonstrates how practical agent systems can be developed using high-level languages like Golog or IndiGolog. Second, it provides BDI languages a clear classical-logic-based semantics and a powerful logical foundation for incorporating new reasoning capabilities not present in typical BDI systems.
\end{abstract}

\section{Introduction}

BDI (Belief-Desire-Intention) agent programming languages and platforms (e.g., PRS [13], AgentSpeak and Jason [22,3], Jack [5], and JADEX [20]) and the situation calculus-based Golog high-level programming language and its successors (e.g., ConGolog [8], IndiGolog [26,9], and FLUX [28]) are two of the most well developed approaches within the agent-oriented programming paradigm. In this paper, we analyze the relationship between these two families of languages and show that BDI agent programming languages are closely related to IndiGolog, a situation calculus based programming language supporting online execution of programs in dynamic environments, sensing actions to acquire information from the environment, and exogenous events.

BDI agent programming languages were conceived as a simplified and operationalized version of the BDI (Belief, Desire, Intention) model of agency, which is rooted in philosophical work such as Bratman's [4] theory of practical reasoning and Dennett's theory of intentional systems [10]. Practical work in the area has sought to develop programming languages that incorporate a simplified BDI semantics basis that has a computational interpretation. An important feature of BDI-style programming languages and platforms is their interleaved account of sensing, deliberation, and execution [21, 24]. In BDI systems, abstract plans written by programmers are combined and executed in real-time. By executing as they reason, BDI agents reduce the likelihood that decisions will be made on the basis of outdated beliefs and remain responsive to the environment by making adjustments in the steps chosen as they proceed. Because of this, 
BDI agent programming languages are well suited to implementing systems that need to operate in "soft" real-time scenarios $[18,2,1]$. Unlike in classical planning-based architectures, execution happens at each step. The assumption is that the careful crafting of plans' preconditions to ensure the selection of appropriate plans at execution time, together with a built-in mechanism for retrying alternative options, will usually ensure that a successful execution is found, even in the context of a changing environment.

In contrast to this, high-level programming languages in the Golog line aim for a middle ground between classical planning and normal programming. The idea is that the programmer may write a sketchy non-deterministic program involving domain specific actions and test conditions and that the interpreter will reason about these and search for a valid execution. The semantics of these languages is defined on top of the situation calculus, a popular predicate logic framework for reasoning about action [19, 25]. The interpreter for the language uses an action theory representing the agent's beliefs about the state of the environment and the preconditions and effects of the actions to find a provably correct execution of the program. By controlling the amount of nondeterminism in the program, the high-level program execution task can be made as hard as classical planning or as easy as deterministic program execution. In IndiGolog, this framework is generalized to allow the programmer to control planning/lookahead and support on-line execution and sensing the environment.

In this paper, we show how a BDI agent can be built within the IndiGolog situation calculus-based programming framework. More concretely, we describe how to translate an agent programmed in a typical BDI programming language into a highlevel IndiGolog program with an associated situation calculus action theory, such that (i) their ultimate behavior coincide; and (ii) the original structure of the propositional attitudes (beliefs, intentions, goals, etc.) of the BDI agent and the model of execution are preserved in the IndiGolog translation. We first do this (Section 3) for what we call the core engine of BDI systems, namely, the reactive context-sensitive expansion of events/goals. After this, in Section 4, we show how to accommodate more sophisticated BDI reasoning mechanisms such as goal failure recovery. Before presenting these results, in Section 2, we give a brief overview of BDI programming languages and Gologrelated programming languages. The paper ends with a short discussion in Section 5, where we mention some potential advantages of programming BDI agents in the situation calculus, in particular, various reasoning about action techniques that IndiGolog BDI agents could incorporate.

\section{Preliminaries}

\subsection{BDI Programming}

BDI agent systems were developed as a way of enabling abstract plans written by programmers to be combined and used in real-time, in a way that is both flexible and robust. A BDI system responds to events, the inputs to the system, by selecting a plan from the plan library, and placing it into the intention base, thus committing to the plan for responding to the event/goal in question. The execution of this plan-strategy may, in turn, post new subgoal events to be achieved. The plan library stands for a collection 


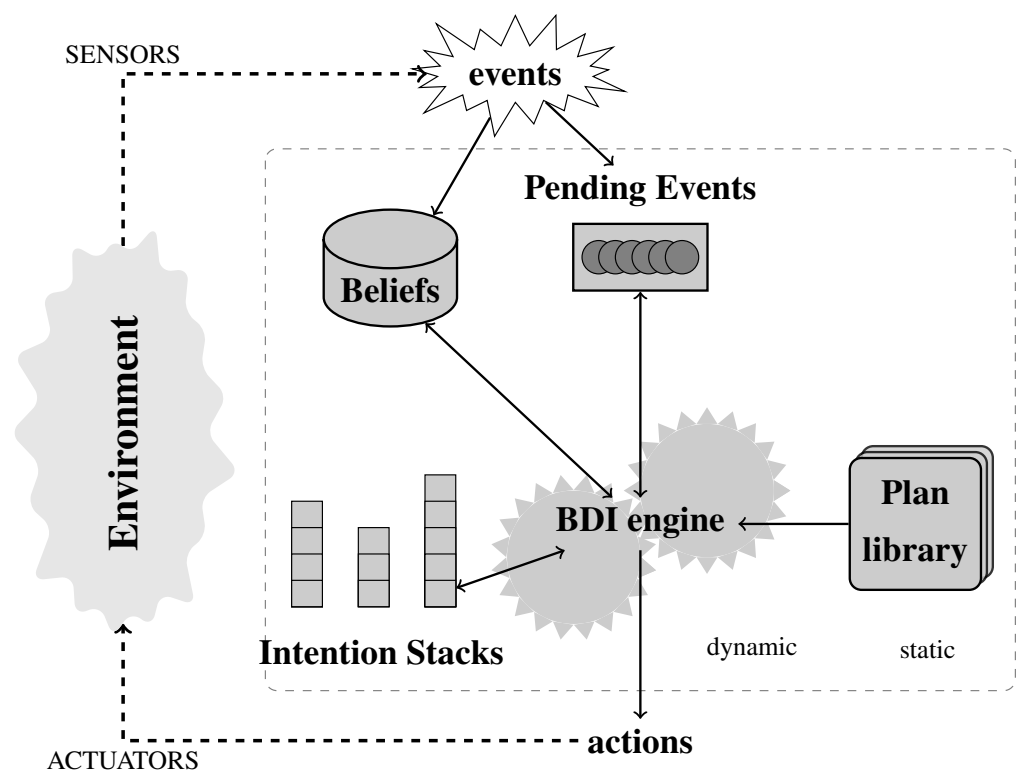

Fig. 1. A typical BDI-style architecture

of pre-defined hierarchical plans indexed by goals (i.e., events) and representing the standard operations in the domain. Figure 1 depicts a typical BDI-style architecture.

There are a number of agent programming languages and development platforms in the BDI tradition, such as PRS [13], AgentSpeak and Jason [22,3], Jack [5], JADEX [20], and 3APL/2APL $[15,7]$. Our discussion is based on the CAN family of BDI languages [29,27] (Conceptual Agent Notation), which are AgentSpeak-like languages with a semantics capturing the common essence of typical BDI systems.

A BDI agent configuration (or simply a BDI agent) $\Upsilon$ is a tuple $\langle\Pi, \mathcal{B}, \mathcal{A}, \Gamma\rangle$, where $\mathcal{B}$ stands for the agent's current beliefs about the world, generally a set of atoms, $\Pi$ is the (static) plan-library, $\mathcal{A}$ is the sequence of actions executed so far, and $\Gamma$ is the multiset of intentions the agent is currently pursuing. The plan library contains plan rules of the form $e: \psi \leftarrow P$, where $e$ is an event/goal that triggers the plan, $\psi$ is the context for which the plan may be applied (i.e., the precondition of the rule), and $P$ is the body of the plan rule $-P$ is a reasonable strategy in order to resolve the event/goal e when condition $\psi$ is believed to hold. The plan-body $P$ is a program built from primitive actions $A$ that the agent can execute directly (e.g., drive $(l o c 1, l o c 3)$ ), operations to add $+b$ and delete $-b$ beliefs, tests for conditions ? $\phi$, and (internal) subgoaling event posting !e (e.g., !Travel (mel, yyz)). Complex plan bodies are built with the usual sequence ; and concurrency $\|$ constructs. There are also a number of auxiliary constructs internally used when assigning semantics to programs: the empty (terminating) program nil; the construct $P_{1} \triangleright P_{2}$, which tries to execute $P_{1}$, falling back to $P_{2}$ if $P_{1}$ is not executable; and $\left(\psi_{1}: P_{1}, \ldots, \psi_{n}: P_{n}\right)$, which encodes a set of alternative guarded plans. Lastly, 
the intention base $\Gamma$ contains the current, partially instantiated, plan-body programs that the agent has already committed to for handling some events-since $\Gamma$ is a multiset it may contain a program more than once.

As with most BDI agent programming languages, the Plotkin-style operational semantics of CAN closely follows Rao and Georgeff's abstract interpreter for intelligent rational agents [24]: (i) incorporate any pending external events; (ii) select an intention and execute a step; and (iii) update the set of goals and intentions. A transition relation $C \longrightarrow C^{\prime}$, on so-called configurations is defined by a set of derivation rules and specifies that executing configuration $C$ a single step yields configuration $C^{\prime}$. A derivation rule consists of a, possibly empty, set of premises, typically involving the existence of transitions together with some auxiliary conditions, and a single transition conclusion derivable from these premises. Two transition relations are used to define the semantics of the CAN language. The first transition relation $\longrightarrow$ defines what it means to execute a single intention and is defined in terms of intention-level configurations of the form $\langle\Pi, \mathcal{B}, \mathcal{A}, P\rangle$ consisting of the agent's plan-library $\Pi$ and belief base $\mathcal{B}$, the actions $\mathcal{A}$ executed so far, and the program $P$ being executed. The second transition relation $\Longrightarrow$ is defined in terms of the first and characterizes what it means to execute a whole agent.

So, the following are some of the intention-level derivation rules for the language: ${ }^{3}$

$$
\begin{gathered}
\frac{\Delta=\{\psi: P \mid e: \psi \leftarrow P \in \Pi\}}{\langle\Pi, \mathcal{B}, \mathcal{A}, ! e\rangle \longrightarrow\langle\Pi, \mathcal{B}, \mathcal{A},| \Delta)\rangle} E v \frac{\left\langle\Pi, \mathcal{B}, \mathcal{A}, P_{1}\right\rangle \longrightarrow\left\langle\Pi, \mathcal{B}^{\prime}, \mathcal{A}^{\prime}, P_{1}^{\prime}\right\rangle}{\left\langle\Pi, \mathcal{B}, \mathcal{A}, P_{1} \triangleright P_{2}\right\rangle \longrightarrow\left\langle\Pi, \mathcal{B}^{\prime}, \mathcal{A}^{\prime}, P_{1}^{\prime} \triangleright P_{2}\right\rangle} \triangleright \\
\frac{\mathcal{B} \models \phi \theta}{\langle\mathcal{B}, \mathcal{A}, ? \phi\rangle \longrightarrow\langle\mathcal{B}, \mathcal{A}, \text { nil }\rangle} ? \frac{\psi: P \in \Delta \quad \mathcal{B}=\psi \theta}{\langle\Pi, \mathcal{B}, \mathcal{A},(\Delta)\rangle \longrightarrow\langle\Pi, \mathcal{B}, \mathcal{A}, P \theta \triangleright(\Delta \backslash\{\psi: P\})\rangle} \text { Sel }
\end{gathered}
$$

Derivation rule $E v$ captures the first stage in the plan selection process for a (pending) event/goal $e$, in which the agent collects, from the plan library, the set $(\Delta)$ of the socalled "relevant" (guarded) plans that may be used to resolve the pending event. Such set is later used by rules $\mathrm{Sel}$ and $\triangleright$ to commit to and execute, respectively, an applicable strategy/plan $P$ (one whose condition $\psi$ is believed true). Notice in rule $\mathrm{Sel}$ how the remaining non-selected plans are kept as backup plans as the second program in the $\square$ construct. Finally, rule? accounts for transitions over a basic test program.

On top of these intention-level derivation rules, the set of agent-level derivation rules are defined. Basically, an agent transition involves either assimilating external events from the environment or executing an active intention. The set of external events $\mathcal{E}$ stands for those external events that were "sensed" by the agent, and it may include external achievement events of the form ! $e$ as well as belief update events of the form $+b$ and $-b$. Also, in the rules below, the following auxiliary function is used to represent the set of achievement events caused by belief changes: $\Omega\left(\mathcal{B}, \mathcal{B}^{\prime}\right)=\left\{! b^{-} \mid \mathcal{B} \models b, \mathcal{B}^{\prime} \not=\right.$

\footnotetext{
${ }^{3}$ Configurations must also include a variable substitution $\theta$ for keeping track of all bindings done so far during the execution of a plan-body. For legibility, we keep substitutions implicit in places where they need to be carried across multiple rules (e.g., in rule ?).
} 


$$
\begin{aligned}
& b\} \cup\left\{! b^{+}|\mathcal{B}| \models b, \mathcal{B}^{\prime}=b\right\} . \\
& \frac{\mathcal{E} \text { is a set of external events } \quad \mathcal{B}^{\prime}=(\mathcal{B} \backslash\{b \mid-b \in \mathcal{E}\}) \cup\{b \mid+b \in \mathcal{E}\}}{\langle\Pi, \mathcal{B}, \mathcal{A}, \Gamma\rangle \Longrightarrow\left\langle\Pi, \mathcal{B}^{\prime}, \mathcal{A}, \Gamma \uplus\{! e \mid ! e \in \mathcal{E}\} \uplus \Omega\left(\mathcal{B}, \mathcal{B}^{\prime}\right)\right\rangle} A_{\text {ext }} \\
& \frac{P \in \Gamma\langle\Pi, \mathcal{B}, \mathcal{A}, P\rangle \longrightarrow\left\langle\Pi, \mathcal{B}^{\prime}, \mathcal{A}^{\prime}, P^{\prime}\right\rangle}{\langle\Pi, \mathcal{B}, \mathcal{A}, \Gamma\rangle \Longrightarrow\left\langle\Pi, \mathcal{B}^{\prime}, \mathcal{A}^{\prime},(\Gamma \backslash\{P\}) \uplus\left\{P^{\prime}\right\} \uplus \Omega\left(\mathcal{B}, \mathcal{B}^{\prime}\right)\right\rangle} A_{\text {exec }}
\end{aligned}
$$

Rule $A_{e x t}$ assimilates a set of external events, including achievements events of the form !e, as well as belief update events of the form $+b$ and $-b$, after which both belief and intention bases of the agent may be updated. Note that, by means of auxiliary function $\Omega$, a new (achievement) event of the form $! b^{+}$or $! b^{-}$is posted for each belief $b$ that changes due to an external belief update; such an event may in turn trigger some new behavior.

Rule $A_{\text {exec }}$ states that the agent may evolve one step if an active intention $P$ can be advanced one step with remaining intention $P^{\prime}$ being left to execute. In such a case, the intention base is updated by replacing $P$ with $P^{\prime}$ and including the belief update events produced by potential changes in the belief base. Observe that the intention base is a multi-set, which means that it may contain several occurrences of the same intention.

Relative to the above derivation rules, one can formally define the meaning of an agent as its possible execution traces. (See $[29,27]$ for the complete semantics.)

Definition 1 (BDI Execution). A BDI execution $E$ of an agent $\Upsilon_{0}=\left\langle\Pi, \mathcal{B}_{0}, \mathcal{A}_{0}, \Gamma_{0}\right\rangle$ is a, possibly infinite, sequence of agent configurations $\Upsilon_{0} \cdot \Upsilon_{1} \cdot \ldots \cdot \Upsilon_{n} \cdot \ldots$ such that $\Upsilon_{i} \Longrightarrow \Upsilon_{i+1}$, for all $i \geq 0$.

\subsection{High-Level Programming in Golog}

The situation calculus $[19,25]$ is a logical language specifically designed for representing dynamically changing worlds in which all changes are the result of named actions The constant $S_{0}$ is used to denote the initial situation where no actions have yet been performed. Sequences of actions are built using the function symbol $d o: d o(a, s)$ denotes the successor situation resulting from performing action $a$ in situation $s$. Relations whose truth values vary from situation to situation are called fluents, and are denoted by predicate symbols taking a situation term as their last argument (e.g., $\operatorname{Holding}(x, s))$. A special predicate $\operatorname{Poss}(a, s)$ is used to state that action $a$ is executable in $s$.

Within this language, we can formulate action theories describing how the world changes as the result of the available actions. For example, a basic action theory [25] includes domain-independent foundational axioms to describe the structure of situations, one successor state axiom per fluent (capturing the effects and non-effects of actions), one precondition axiom per action (specifying when the action is executable), and initial state axioms describing what is true initially (i.e., what is true in the initial situation $S_{0}$ ).

On top of situation calculus action theories, logic-based programming languages can be defined, which, in addition to the primitive actions, allow the definition of complex actions. Golog [17], the first situation calculus-based agent programming language, provides all the usual control structures (e.g., sequence, iteration, conditional, 
etc.) plus some nondeterministic constructs. These nondeterministic constructs allow the loose specification of programs by leaving "gaps" that ought to be resolved by the reasoner/planner or executor. ConGolog [8] extends Golog to support concurrency. To provide an intuitive overview of the language, consider the following nondeterministic program for an agent that goes to work in the morning: ${ }^{4}$

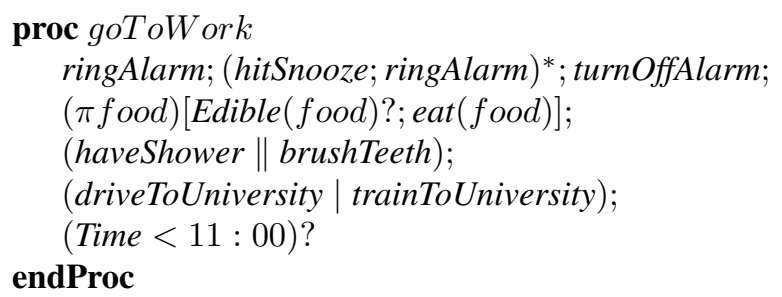

While this high-level program provides a general strategy for getting up and going to work, it is underspecified, and many details, such as what to eat and how to travel to work, are left open. Program $\delta_{1} \mid \delta_{2}$ nondeterministically chooses between programs $\delta_{1}$ and $\delta_{2}, \pi x . \delta(x)$ executes program $\delta(x)$ for some legal binding for variable $x$, and $\delta^{*}$ performs $\delta$ zero or more times. Concurrency is supported by the following three constructs: $\left(\delta_{1} \| \delta_{2}\right)$ expresses the concurrent execution (interpreted as interleaving) of programs $\delta_{1}$ and $\left.\left.\delta_{2} ; \delta_{1}\right\rangle\right\rangle \delta_{2}$ expresses the concurrent execution of $\delta_{1}$ and $\delta_{2}$ with $\delta_{1}$ having higher priority; and $\delta^{\|}$executes $\delta$ zero or more times concurrently. Note that a concurrent process may become (temporarily) blocked when it reaches a test/wait action $\phi$ ? whose condition $\phi$ is false, or a primitive action whose preconditions are false. Test/wait actions can also be used to control which nondeterministic branches can be executed, e.g. $\left[\left(\phi ? ; \delta_{1}\right) \mid\left(\neg \phi ? ; \delta_{2}\right)\right]$, and to constrain the value of a nondeterministically bound variable, e.g., $\pi x .[\phi(x) ? ; \delta(x)]$. Finally, the language also accommodates the standard if-then-elses, while loops, and recursive procedures.

Finding a legal execution of a high-level program is at the core of the whole approach. Originally, Golog and ConGolog programs were intended to be executed offline, that is, a complete execution was obtained before committing even to the first action. However, IndiGolog [26,9], the latest language within the Golog family, provides a formal logic-based account of interleaved planning, sensing, and action by executing programs online and using a specialized new construct $\Sigma(\delta)$, the search operator, to perform local offline planning when required.

Roughly speaking, an online execution of a program finds a next possible action, executes it in the real world, then obtains sensing results and observed exogenous actions, and repeats the cycle until the program's execution is completed.

Formally, an online execution is a sequence of so-called online configuration of the form $(\delta, \sigma)$, where $\delta$ is a high-level program and $\sigma$ is a history (see [9] for its formal definition). A history contains the sequence of actions executed so far as well as the sensing information obtained. Online executions are characterized in terms of the following two predicates [8]: $\operatorname{Final}(\delta, s)$ holds if program $\delta$ may legally terminate in situation $s$; and $\operatorname{Trans}\left(\delta, s, \delta^{\prime}, s^{\prime}\right)$ holds if a single step of program $\delta$ in situation $s$

\footnotetext{
${ }^{4}$ We thank Ryan Kelly and Adrian Pearce for allowing us to re-use their example.
} 
may lead to situation $s^{\prime}$ with $\delta^{\prime}$ remaining to be executed. In the next section, we will generalize the notion of online execution to suit our purposes.

\section{BDI Programming in IndiGolog}

Programming a BDI agent in the situation calculus amounts to developing a special basic action theory and a special IndiGolog high-level agent program to be executed with it. From now on, let $\Upsilon=\langle\Pi, \mathcal{B}, \mathcal{A}, \Gamma\rangle$ be the BDI agent to program in IndiGolog.

\subsection{The BDI Basic Action Theory}

We start by showing how to obtain an action theory $\mathcal{D}^{\Upsilon}$ for our agent $\Upsilon$. We assume that $\Upsilon$ is stated over a first-order language $\mathcal{L}_{B D I}$ containing finitely many belief and event atomic relations, namely, $b_{1}\left(\boldsymbol{x}_{\mathbf{1}}\right), \ldots, b_{n}\left(\boldsymbol{x}_{\boldsymbol{n}}\right)$ and $e_{1}\left(\boldsymbol{x}_{\mathbf{1}}\right), \ldots, e_{m}\left(\boldsymbol{x}_{\boldsymbol{n}}\right)$.

Let us then define what the fluents and actions available in the situation calculus language $\mathcal{L}_{\text {sitCalc }}$ are. First, for every belief atomic predicate $b(\boldsymbol{x})$ in $\mathcal{L}_{B D I}$, the language $\mathcal{L}_{\text {sitCalc }}$ includes a relational fluent $b(\boldsymbol{x}, s)$ together with two primitive actions $\operatorname{add}_{b}(\boldsymbol{x})$ and $\operatorname{del}_{b}(\boldsymbol{x})$ which are meant to change the fluent's truth value. Second, for each achievement event type $e(\boldsymbol{x})$ in the domain, there is a corresponding action term $\operatorname{ach}_{e}(\boldsymbol{x})$ in $\mathcal{L}_{\text {sitCalc }}$. Finally, for every action atom $A(\boldsymbol{x})$ in $\mathcal{L}_{B D I}$, there is a corresponding action function $A(\boldsymbol{x})$ in $\mathcal{L}_{\text {sitCalc }}$.

In addition, the language $\mathcal{L}_{\text {sitCalc }}$ shall include one auxiliary distinguished fluent and two actions to model external event handling. Fluent PendingEv $(s)$ stands for the multi-set of events that are "pending" and need to be handled, either belief update or achievement events. This fluent is affected by two actions. Whereas action post $(e)$ indicates the external posting of event $e$; action serve $(e)$ indicates that (pending) event $e$ has been selected and is being handled. In both actions, argument $e$ is of sort action.

Let us now construct the basic action theory $\mathcal{D}^{\Upsilon}$ corresponding to a BDI agent $\Upsilon=\langle\Pi, \mathcal{B}, \mathcal{A}, \Gamma\rangle$, as follows:

1. The initial description in $\mathcal{D}^{\Upsilon}$ is defined in the following way:

$$
\begin{gathered}
\mathcal{D}_{S_{0}}^{\Upsilon}=\bigcup_{i=1}^{n}\left\{\forall \boldsymbol{x} . b_{i}\left(\boldsymbol{x}, S_{0}\right) \equiv \boldsymbol{x}=\boldsymbol{t}_{\boldsymbol{i}}^{\mathbf{1}} \vee \ldots \vee \boldsymbol{x}=\boldsymbol{t}_{\boldsymbol{i}}^{\boldsymbol{k}_{\boldsymbol{i}}}\right\} \cup \\
\left\{\forall a \cdot \operatorname{Exog}(a) \equiv\left(\exists a^{\prime}\right) a=\operatorname{post}\left(a^{\prime}\right)\right\},
\end{gathered}
$$

where for every $i \in\{1, \ldots, n\}, \mathcal{B} \models b_{i}(\boldsymbol{x}) \equiv\left[\boldsymbol{x}=\boldsymbol{t}_{\boldsymbol{i}}^{\mathbf{1}} \vee \ldots \vee \boldsymbol{x}=\boldsymbol{t}_{\boldsymbol{i}}^{\boldsymbol{k}_{\boldsymbol{i}}}\right]$, for some $k_{i} \geq 0-b_{i}\left(\boldsymbol{t}_{\boldsymbol{i}}^{\mathbf{1}}\right), \ldots, b_{i}\left(\boldsymbol{t}_{\boldsymbol{i}}^{\boldsymbol{k}_{\boldsymbol{i}}}\right)$ are all the true belief atoms in $\mathcal{B}$ with respect to belief relation $b_{i}$ (each $\boldsymbol{t}_{\boldsymbol{i}}^{\boldsymbol{j}}$ is a vector of ground terms).

2. The following precondition axioms, for every fluent $b(\boldsymbol{x})$ and action type $A(\boldsymbol{x})$ :

$$
\begin{array}{lr}
\operatorname{Poss}(\operatorname{serve}(a), s) \equiv(a \in \operatorname{PendingEv}(s)) & \operatorname{Poss}(A(\boldsymbol{x}), s) \equiv \text { True } \\
\operatorname{Poss}\left(\operatorname{add}_{b}(\boldsymbol{x}), s\right) \equiv \operatorname{Poss}\left(\operatorname{del}_{b}(\boldsymbol{x}), s\right) \equiv \text { True } & \operatorname{Poss}(\operatorname{post}(a), s) \equiv \text { True }
\end{array}
$$

3. For every domain fluent $b(\boldsymbol{x}, s), \mathcal{D}^{\Upsilon}$ includes the following successor state axiom:

$$
\begin{aligned}
& b(\boldsymbol{x}, \operatorname{do}(a, s)) \equiv \\
& \quad a=a d d_{b}(\boldsymbol{x}) \vee a=\operatorname{post}\left(\operatorname{add}_{b}(\boldsymbol{x})\right) \vee b(\boldsymbol{x}, s) \wedge\left(a \neq \operatorname{del}_{b}(\boldsymbol{x}) \wedge a \neq \operatorname{post}\left(\operatorname{del}_{b}(\boldsymbol{x})\right) .\right.
\end{aligned}
$$


That is, the truth-value of fluent $b$ is affected only by actions $a d d_{b}$ and $d e l_{b}$, either internally executed or externally sensed from the environment.

More importantly, action theory $\mathcal{D}^{\Upsilon}$ includes a successor state axiom for fluent PendingEv $(\operatorname{do}(a, s))$ specifying how the multi-set of pending events changes:

PendingEv $(\operatorname{do}(a, s))=v \equiv\left[\gamma(a, v, s) \vee\right.$ PendingEv $\left.(s)=v \wedge \neg \exists v^{\prime} \cdot \gamma\left(a, v^{\prime}, s\right)\right]$;

where:

$$
\begin{aligned}
& \gamma(a, v, s) \stackrel{\text { def }}{=}\left(\bigvee_{i=1}^{n}\left[\gamma_{i}^{+}(a, v, s) \vee \gamma_{i}^{-}(a, v, s)\right] \vee \bigvee_{i=1}^{m}\left[\gamma_{i}^{e}(a, v, s)\right] \vee\right. \\
& \left.\quad \exists a^{\prime} . a=\operatorname{serve}\left(a^{\prime}\right) \wedge v=\operatorname{PendingEv}(s) \backslash\left\{a^{\prime}\right\}\right) ;
\end{aligned}
$$

That is, an actual change in the belief of an atom, either due to the execution of some intention or an external event, automatically produces a corresponding pending belief update event. Moreover, an external achievement event $a c h_{e}(\boldsymbol{x})$ becomes pending when sensed. On the other hand, an event $e$ ceases to be pending when action serve $(e)$ is executed.

4. Theory $\mathcal{D}^{\Upsilon}$ includes unique name axioms for all actions in $\mathcal{L}_{\text {sitCalc }}$, as well as the standard domain-independent foundational axioms for the situation calculus ([25]).

This concludes the construction of the BDI basic action theory $\mathcal{D}^{\Upsilon}$.

\subsection{The BDI Agent Program}

Let us now construct the IndiGolog BDI agent program $\delta^{\Upsilon}$ that is meant to execute relative to the BDI action theory $\mathcal{D}^{\Upsilon}$. We start by showing how to inductively transform a BDI plan-body program $P$ into an IndiGolog program $\delta_{P}$, namely (remember that plan-bodies programs are used to build BDI plans in the plan library):

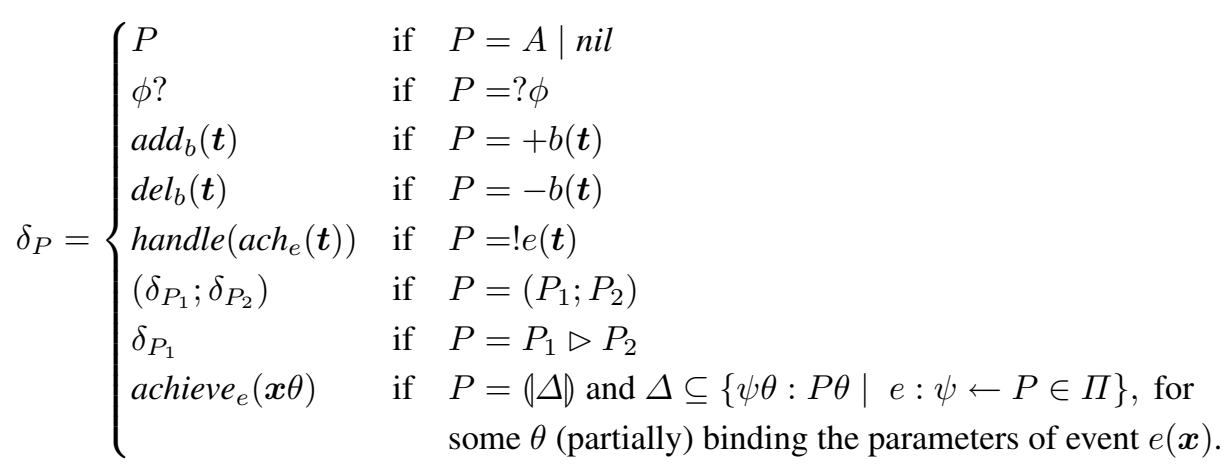

Notice that achievement events !e occurring in a plan are handled via simple plan invocation, by invoking procedure handle. Also, for now, the translation just ignores the 
second program in $P_{1} \triangleright P_{2}$, as the version of CAN we consider in this section does not try $P_{2}$ when $P_{1}$ happens to fail. We shall revisit this later in Section 4. Finally, when the BDI program is a set of (relevant) alternatives of the form $(\Delta)$ for some event $e$, we map it to a procedure call that will basically amount to a non-deterministic choice among all such alternatives (see below).

Next, we describe how to transform the BDI plans in the agent's plan library. To that end, suppose that $e(\boldsymbol{x})$ is an event in the BDI language $\mathcal{L}_{B D I}$ such with the following $n \geq 0$ plans in $\Pi\left(\boldsymbol{v}_{\boldsymbol{t}}\right.$ denotes all the distinct free variables in the terms $\left.\boldsymbol{t}\right)$ :

$$
e\left(\boldsymbol{t}_{\boldsymbol{i}}\right): \psi_{i}\left(\boldsymbol{v}_{\boldsymbol{t}_{\boldsymbol{i}}}, \boldsymbol{y}_{\boldsymbol{i}}\right) \leftarrow P_{i}\left(\boldsymbol{v}_{\boldsymbol{t}_{\boldsymbol{i}}}, \boldsymbol{y}_{\boldsymbol{i}}, \boldsymbol{z}_{\boldsymbol{i}}\right) \text {, where } i \in\{1, \ldots, n\} \text {. }
$$

Then, we build the following high-level Golog procedure with $n$ non-deterministic choices (i.e., as many as plan-rules for the event):

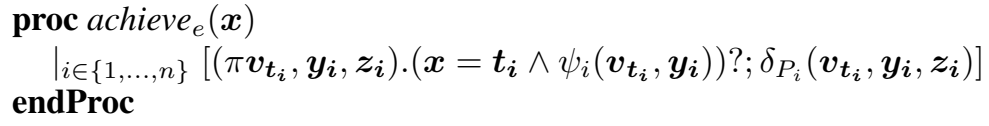

Roughly speaking, executing achieve $_{e}(\boldsymbol{x})$ involves nondeterministically choosing among the $n$ available options in the plan library for event $e$. See that the first test statement in each option amounts to checking the relevance and applicability of the option. Thus, the execution of achieve $_{e}(\boldsymbol{x})$ is bound to block if no option is relevant or applicable. In particular, the procedure will always block if the agent $\Upsilon$ has no plan to handle the event in question-that is, if $n=0$, the corresponding Golog procedure is simply?(False).

Let $\Delta_{\Pi}$ denote the set of Golog procedures as above, one per event in the BDI language, together with the following procedure:

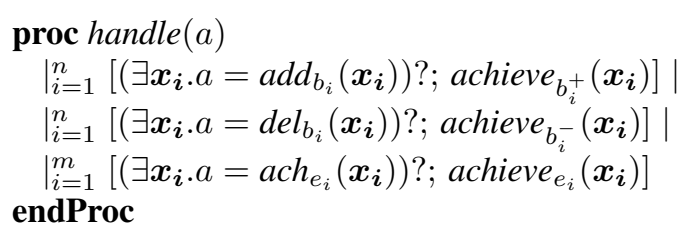

That is, when $a$ is a legal event (belief update or achievement goal), procedure handle $(a)$ calls the appropriate procedure that is meant to resolve the event. Observe that this program contains two nondeterministic programs per belief atom in the domain (one to handle its addition and one to handle its deletion from the belief base), plus one nondeterministic program per achievement event in the domain.

Finally, we define the top-level IndiGolog BDI agent program as follows:

$$
\delta^{\Upsilon \stackrel{\text { def }}{=}} \Delta_{\Pi} ;\left[\delta_{\text {env }} \|\left(\delta_{\Gamma} \| \delta_{B D I}\right)\right] ;(\neg \exists e \operatorname{Pending} E v(e)) ?,
$$

where (assuming that $\Gamma=\left\{P_{1}, \ldots, P_{n}\right\}$ ):

$\delta_{\Gamma} \stackrel{\text { def }}{=} \delta_{P_{1}}\|\cdots\| \delta_{P_{n}} ; \quad \delta_{\text {env }} \stackrel{\text { def }}{=}(\pi a . \operatorname{Exog}(a) ? ; a)^{*} ; \quad \delta_{B D I} \stackrel{\text { def }}{=}[\pi a . \operatorname{serve}(a) ; \operatorname{handle}(a)]^{\|}$.

The set of programs $\Delta_{\Pi}$ provides the environment encoding the BDI plan library. Program $\delta_{\Gamma}$ accounts for all current intentions in $\Upsilon$; if $\Gamma=\emptyset$, then $\delta_{\Gamma}=$ nil. In 
turn, program $\delta_{\text {env }}$ models the external environment, which can perform zero, one, or more actions of the form post $(a)$, representing an external achievement event goal $\left(a=a_{c h}(\boldsymbol{t})\right)$ or a belief update event $\left(a=a d d_{b}(\boldsymbol{t})\right.$ or $\left.a=\operatorname{del}_{b}(\boldsymbol{t})\right)$.

The most interesting part of $\delta^{\Upsilon}$ is indeed the ConGolog program $\delta_{B D I}$, which implements (part of) the BDI execution cycle. More concretely, $\delta_{B D I}$ is responsible for selecting an external event and spawning a new "intention" concurrent thread for handling it. To that end, $\delta_{B D I}$ picks an event $a$ (e.g., $a d d_{A t}(23,32)$ or achieve $_{\text {moveTo }}(0,0)$ ) to be served and executes action serve $(a)$. Observe that an event can be served only if it is currently pending (see action precondition for action serve (a) in Subsection 3.1). After the action serve $(a)$ has been successfully executed, the selected event $a$ is actually handled, by calling procedure handle $(a)$ defined in $\Delta_{\Pi}$. More importantly, this is done in a "new" concurrent thread, so that program $\delta_{B D I}$ is still able to serve and handle other pending events. The use of concurrent iteration to spawn a new intention from the "main BDI thread" is inspired from the server example application in [8].

Note that $\Delta_{\Pi}$ and $\delta_{\Gamma}$ are domain dependent, i.e., they are built relative to a particular BDI agent $\Upsilon$, whereas programs $\delta_{B D I}$ and $\delta_{e n v}$ are independent of the BDI agent being encoded. Observe also that the whole high-level program $\delta^{\Upsilon}$ may terminate only when no more events are pending.

From now on, let $\mathcal{G}^{\Upsilon}=\left\langle\mathcal{D}^{\Upsilon}, \delta^{\Upsilon}, \mathcal{A}\right\rangle$ denote the IndiGolog agent for BDI agent $\Upsilon$.

\subsection{LC-Online Executions}

Once we have a BDI IndiGolog program $\mathcal{G}^{\Upsilon}$ on hand, we should be able to execute it and obtain the same behavior and outputs as with the original BDI agent. Unfortunately, we cannot execute $\mathcal{G}^{\Upsilon}$ online, as defined in [9], as such executions may commit too early to free variables in a program-online executions are sequences of ground online configurations. For example, program $\pi$ x.turnOffAlarm;Edible $(x)$ ?; eat $(x)$ may do a transition by execution the primitive action turnOffAlarm and instantiating $x$ to Clock, yielding remaining program Edible(Clock)?; eat(Clock), which is bound to fail as the object picked is not edible. In fact, as no constraints are imposed on $x$ in the first transition, any binding for it would be legal.

What we need, instead, is an account of execution that commits to free variables only when necessary. To that end, we generalize the online execution notion from $[9,26]$ to what we call least-committed online executions. Below, we use end $[\sigma]$ to denote the situation term corresponding to the history $\sigma$; and Axioms $[\mathcal{D}, \sigma]$ to denote the complete set of axioms in the IndiGolog theory, which includes the action theory $\mathcal{D}$ for the domain, the sensing results gathered so far in history $\sigma$, and all axioms for Trans and Final. So, we first define two meta-theoretic versions of relations Trans and Final as follows:

$$
\begin{aligned}
& \operatorname{m\operatorname {Trans}}\left(\delta(\boldsymbol{x}, \boldsymbol{y}), \sigma, \delta^{\prime}(\boldsymbol{x}, \boldsymbol{z}), \sigma^{\prime}\right) \stackrel{\text { def }}{=} \\
& \quad \operatorname{Axioms}[\mathcal{D}, \sigma] \models \exists \boldsymbol{y} \forall \boldsymbol{x}, \boldsymbol{z} . \operatorname{Trans}\left(\delta(\boldsymbol{x}, \boldsymbol{y}), \text { end }[\sigma], \delta^{\prime}(\boldsymbol{x}, \boldsymbol{z}), \text { end }\left[\sigma^{\prime}\right]\right) ; \\
& m \operatorname{Final}(\delta(\boldsymbol{x}, \boldsymbol{y}), \sigma) \stackrel{\text { def }}{=} \operatorname{Axioms}[\mathcal{D}, \sigma] \models \exists \boldsymbol{x} . \operatorname{Final}(\delta(\boldsymbol{x}), \text { end }[\sigma]) .
\end{aligned}
$$

Here, $\delta(\boldsymbol{x})$ means that the vector of variables $\boldsymbol{x}$ contains all the free variables mentioned in the program; different variables vectors are assumed disjoint. Thus, $\boldsymbol{x}$ are the 
free variables in $\delta$ that are still free in $\delta^{\prime} ; \boldsymbol{y}$ are the free variables in $\delta$ that have been instantiated and are not present in $\delta^{\prime}$; and $z$ are the new free variables in $\delta^{\prime}$ that did not appear in $\delta$.

We can then define least-committed executions as follows.

Definition 2 (LC-Online Execution). A least-committed online (lc-online) execution of an IndiGolog program $\delta$ starting from a history $\sigma$ is a, possibly infinite, sequence of configurations $\left(\delta_{0}=\delta, \sigma_{0}=\sigma\right),\left(\delta_{1}, \sigma_{1}\right), \ldots$ such that for every $i \geq 0$ :

1. $m \operatorname{Trans}\left(\delta_{i}, \sigma_{i}, \delta_{i+1}, \sigma_{i+1}\right)$ holds; and

2. for all $\delta^{\prime}$ such that $m \operatorname{Trans}\left(\delta_{i}, \sigma_{i}, \delta^{\prime}, \sigma_{i+1}\right)$ and $\delta_{i+1}=\delta^{\prime} \theta$ for some substitution $\theta$, there exists $\theta^{\prime}$ such that $\delta^{\prime}=\delta_{i+1} \theta^{\prime}$.

A finite lc-online execution $\left(\delta_{0}, \sigma_{0}\right), \ldots,\left(\delta_{n}, \sigma_{n}\right)$ is terminating iff $m$ Final $\left(\delta_{n}, \sigma_{n}\right)$ or for all $\delta^{\prime}, \sigma^{\prime} m$ Trans $\left(\delta_{n}, \sigma_{n}, \delta^{\prime}, \sigma^{\prime}\right)$ does not hold.

We notice that, as expected, it can be shown that an lc-online execution stands for all its ground online instances as defined in [9]. However, by executing programs in a least committed way, we avoid premature binding of variables and eliminate some executions where the program is bound to fail.

\subsection{BDI/IndiGolog Bisimulation}

We are now ready to provide the main results of the paper. Namely, we show that given any BDI execution of an agent, there exists a matching execution of the corresponding IndiGolog agent, and vice-versa. In addition, the correspondence in the internal structure of the agents is always maintained throughout the executions.

We start by characterizing when a BDI agent and an IndiGolog agent configuration "match." To that end, we shall use relation $\Upsilon \approx \mathcal{G}$, which, intuitively, holds if a BDI agent $\Upsilon$ and an IndiGolog agent $\mathcal{G}$ represent the same (BDI) agent system. Formally, relation $\langle\Pi, \mathcal{B}, \mathcal{A}, \Gamma\rangle \approx\langle\mathcal{D}, \delta, \sigma\rangle$ holds iff

1. $\delta=\Delta_{\Pi} ;\left[\delta_{\text {env }} \|\left(\delta_{\Gamma^{\prime}} \| \delta_{B D I}\right)\right] ; ?(\neg \exists e$ Pending $E v(e))$, for some $\Gamma^{\prime} \subseteq \Gamma$ such that $\Gamma=\Gamma^{\prime} \uplus\{a \mid$ Axioms $[\mathcal{D}, \sigma] \models a \in$ PendingEv $($ end $[\sigma])\}$;

2. $\mathcal{A}$ and $\sigma$ contain the same sequence of domain actions;

3. for every ground belief atom $b: \mathcal{B} \models b$ iff Axioms $[\mathcal{D}, \sigma] \models b[$ end $[\sigma]]$;

4. $\mathcal{D}=\mathcal{D}^{\Upsilon^{\prime}}$, for some $\Upsilon^{\prime}=\left\langle\Pi, \mathcal{B}^{\prime}, \mathcal{A}, \Gamma\right\rangle$.

The first condition states that the IndiGolog program is of the form shown in equation (1) above (see Section 3.2), but where some active intentions may still be "pending." In other words, some intentions in $\Gamma$ that have not yet started execution may not show up yet as concurrent processes in $\delta$, but they are implicitly represented as "pending" in fluent PendingEv $(s)$. The second requirement states that both agents have performed the same sequence of domain primitive actions, that is, actions other than the internal ones serve $(a), \operatorname{post}(a), a d d_{b}(\boldsymbol{x})$, and $\operatorname{del}_{b}(\boldsymbol{x})$. The third condition requires both agents to coincide on what they currently believe. Observe that the initial beliefs of the IndiGolog agent do not necessarily need to coincide with those of the BDI agent, as long as the 
current beliefs do (i.e., the beliefs that hold after history $\sigma$ ); in fact the BDI agent configuration does not specify what it believed initially, while the IndiGolog agent's action theory does. Lastly, the IndiGolog agent executes relative to a basic action theory whose dynamics are as described in Section 3.1.

First of all, it is possible to show that the encoding of initial BDI agents, that is agents that have not yet performed any action, into IndiGolog agents described above is in the $\approx$ relation with the original BDI agent.

Theorem 1. Let $\Upsilon$ be an initial BDI agent (that is, $\mathcal{A}=\epsilon$ ). Then, $\Upsilon \approx\left\langle\mathcal{D}^{\Upsilon}, \delta^{\Upsilon}, \mathcal{A}\right\rangle$.

The importance of a BDI agent and an IndiGolog agent being in the $\approx$ relation is that their respective transitions can then always be simulated by the other type of agent To demonstrate that, we first show that any BDI transition can be replicated by the corresponding IndiGolog agent. Observe that IndiGolog may need several transitions to replicate the BDI transition when it comes to assimilating external events; whereas BDI agents incorporate sets of external events in a single transition, the IndiGolog agent incorporates one event per transition. Also, IndiGolog agents ought to execute the special action serve $(a)$ to start handling external achievement events.

Theorem 2. Let $\Upsilon$ be a BDI agent and $\langle\mathcal{D}, \delta, \sigma\rangle$ an IndiGolog agent such that $\Upsilon \approx$ $\langle\mathcal{D}, \delta, \sigma\rangle$. If $\Upsilon \Longrightarrow \Upsilon^{\prime}$, then there exists a program $\delta^{\prime}$ and a history $\sigma^{\prime}$ such that $m$ Trans $^{*}\left(\delta, \sigma, \delta^{\prime}, \sigma^{\prime}\right)$ holds relative to action theory $\mathcal{D}$, and $\Upsilon^{\prime} \approx\left\langle\mathcal{D}, \delta^{\prime}, \sigma^{\prime}\right\rangle$.

Furthermore, in the other direction, any step in a BDI IndiGolog execution can always be "mimicked" by the corresponding BDI agent.

Theorem 3. Let $\Upsilon$ and $\langle\mathcal{D}, \delta, \sigma\rangle$ be a BDI and an IndiGolog agents, respectively, such that $\Upsilon \approx\langle\mathcal{D}, \delta, \sigma\rangle$. Suppose that $m \operatorname{Trans}\left(\delta, \sigma, \delta^{\prime}, \sigma^{\prime}\right)$ holds relative to action theory $\mathcal{D}$, for some IndiGolog program $\delta^{\prime}$ and history $\sigma^{\prime}$. Then, either $\Upsilon \approx\left\langle\mathcal{D}, \delta^{\prime}, \sigma^{\prime}\right\rangle$ or there exists a BDI agent $\Upsilon^{\prime}$ such that $\Upsilon \Longrightarrow \Upsilon^{\prime}$ and $\Upsilon^{\prime} \approx\left\langle\mathcal{D}, \delta^{\prime}, \sigma^{\prime}\right\rangle$.

So, when the IndiGolog agent performs a transition it remains "equivalent" to the BDI agent or to some evolution of the BDI agent. The former case applies only when the transition in question involved the execution of a serve $(a)$ action to translate a pending event into a concurrent process.

Putting both theorems together, our encoding allows IndiGolog to bisimulate BDI agents.

\section{BDI Failure Handling}

Since BDI systems are meant to operate in dynamic settings, plans that were supposed to work may fail due to changes in the environment. Indeed, a plan may fail because a test condition $? \phi$ is not believed true, an action cannot be executed, or a sub-goal event does not have any applicable plans. The BDI language we have discussed so far has no strategy towards failed plans or intentions, once an intention cannot evolve, it simply remains in the intention base blocked. In this section, we discuss how BDI programming languages typically address plan/intention failure and show how the above IndiGolog 
encoding can be extended accordingly. In particular, we show how agents can abandon failed intentions and recover from problematic plans by trying alternative options.

Before getting into technical details, we shall first introduce a new construct into the IndiGolog language. In "Golog-like" languages, a program that is blocked may not be dropped for the sake of another program. To overcome this, we introduce the construct $\delta_{1} \triangleright \delta_{2}$ with the intending meaning that $\delta_{1}$ should be executed, falling back to $\delta_{2}$ if $\delta_{1}$ becomes blocked: ${ }^{5}$

$$
\begin{aligned}
\operatorname{Trans}\left(\delta_{1} \triangleright \delta_{2}, s, \delta^{\prime}, s^{\prime}\right) \equiv & \left(\exists \gamma \cdot \operatorname{Trans}\left(\delta_{1}, s, \gamma, s^{\prime}\right) \wedge \delta^{\prime}=\gamma \triangleright \delta_{2}\right) \vee \\
& \neg \exists \gamma, s^{\prime \prime} . \operatorname{Trans}\left(\delta_{1}, s, \gamma, s^{\prime \prime}\right) \wedge \operatorname{Trans}\left(\delta_{2}, s, \delta^{\prime}, s^{\prime}\right) ; \\
\operatorname{Final}\left(\delta_{1} \triangleright \delta_{2}, s, \delta^{\prime}, s^{\prime}\right) \equiv & \operatorname{Final}\left(\delta_{1}, s\right) \vee \neg \exists \gamma, s^{\prime \prime} . \operatorname{Trans}\left(\delta_{1}, s, \gamma, s^{\prime \prime}\right) \wedge \operatorname{Final}\left(\delta_{2}, s\right) .
\end{aligned}
$$

\subsection{Dropping Impossible Intentions}

It is generally accepted that intentions that cannot execute further may simply be dropped by the agent - rational agents should not pursue intentions/goals that are deemed impossible $[23,6]$. This is indeed the behavior of AgentSpeak agents. ${ }^{6}$

The BDI language of Section 2.1 can be easily extended to provide such an intentiondropping facility, by just adding the following agent-level operational rule:

$$
\frac{P \in \Gamma \quad\langle\Pi, \mathcal{B}, \mathcal{A}, P\rangle \nrightarrow}{\langle\Pi, \mathcal{B}, \mathcal{A}, \Gamma\rangle \Longrightarrow\langle\Pi, \mathcal{B}, \mathcal{A}, \Gamma \backslash\{P\}\rangle} A_{\text {clean }}
$$

That is, an agent may choose to just drop an intention from its intention base if it cannot execute further in the current mental state. To mimic this behavior in our BDI IndiGolog formalization, we slightly modify the domain-independent program $\delta_{B D I}$ as follows:

$$
\delta_{B D I} \stackrel{\text { def }}{=}[\pi \text { a.serve }(a) ;(\text { handle }(a) \triangleright(\text { True }) ?)]^{\|} .
$$

Here, a pending event is handled within the scope of a $\triangleright$, which basically allows the intention thread to simply terminate if it becomes blocked. Notice that, as with BDI languages, for procedure handle $(a)$ to be blocked, every sub-goal event triggered by the handling of $a$ (represented in the IndiGolog program as simple procedure calls) ought to be blocked. Observe also that in this approach, only the main program corresponding to a top-level event may be dropped, not lower-level instrumental subgoals.

\subsection{BDI Goal Failure Recovery}

Merely dropping a whole intention when it becomes blocked provides a rather weak level of commitment to goals. The failure of a plan should not be equated to the failure of its parent goal, as there could be alternative ways to achieve the latter. For example, suppose an agent has the goal to quench her thirst, and in the service of this goal, she

\footnotetext{
${ }^{5}$ One could easily extend these definitions to only allow dropping a blocked $\delta_{1}$ under given conditions; this could be used to implement "time outs" or allow blocking for synchronization.

${ }^{6}$ There has been work on more sophisticated treatments of plan failure in extensions of AgentSpeak; see for instance [3].
} 
adopts the subgoal of buying a can of soda [27]. However, upon arrival at the store, she realizes that all the cans of soda are sold out. Fortunately though, the shop has bottles of water. In this situation, it is irrational for the agent to drop the whole goal of quenching her thirst just because soda is not available. Yet this is what an AgentSpeak agent would do. Similarly, we do not expect the agent to fanatically insist on her subgoal and just wait indefinitely for soda to be delivered. What we expect is the agent to merely drop her commitment to buy soda and adopt the alternative subgoal of buying a bottle of water, thereby achieving the main goal.

As a matter of fact, one of the typical features of implemented BDI languages is that of plan-goal failure recovery: if a plan happens to fail for a goal, usually due to unexpected changes in the environment, another plan is tried to achieve the goal. If no alternative strategy is available, then the goal is deemed failed and failure is propagated to higher-level motivating goals, and so on. This mechanism thus provides a stronger level of commitment to goals, by decoupling plan failure from goal failure.

To accommodate this approach to failure recovery, we further extend the BDI language of Section 2.1 , by providing the following additional derivation rule for "try" construct $\triangleright$ :

$$
\frac{\left\langle\Pi, \mathcal{B}, \mathcal{A}, P_{1}\right\rangle \nrightarrow\left\langle\Pi, \mathcal{B}^{\prime}, \mathcal{A}^{\prime}, P_{2}^{\prime}\right\rangle \longrightarrow\left\langle\Pi, \mathcal{B}^{\prime}, \mathcal{A}^{\prime}, P_{2}^{\prime}\right\rangle}{\left\langle\Pi, \mathcal{B}, \mathcal{A}, P_{1} \triangleright P_{2}\right\rangle \longrightarrow\left\langle\Pi, \mathcal{B}^{\prime}, \mathcal{A}^{\prime}, P_{2}^{\prime}\right\rangle} \triangleright_{f}
$$

That is, if the current strategy $P_{1}$ is blocked but the alternative backup program $P_{2}$ is able to evolve, then it is legal to drop $P_{1}$ and switch to $P_{2}$. Observe that due to derivation rules $E v$ and $S e l, P_{2}=(\Delta)$ will encode the set of relevant plans that have not yet been tried for the event being addressed. From now on, let the CAN language refer to our extended BDI language, with both new derivation rules $A_{\text {clean }}$ and $\triangleright_{f}$ for failure included.

Hence, due to the interaction between derivation rules $E v, S e l$ and $\triangleright_{f}$, a CAN BDI agent executes a program $\left.P_{1} \triangleright \mid \Delta\right)$ in order to resolve an goal event !e. When the current strategy $P_{1}$ being pursued is not able to make a step, the agent may check the set of alternatives $(\Delta)$ in the hope of finding another option $P_{2}$ for addressing $e$. If one is found, the agent may opt to abandon its strategy $P_{1}$ and continue with $P_{2}$. (Details can be found in $[29,27]$.)

Let us now describe how to replicate this failure recovery behavior within our IndiGolog framework of Section 3. For simplicity, we shall assume that, as with actions, only ground posting of subgoal events are allowed in the BDI language. This means that all variables $\boldsymbol{x}$ in an event posting $! e(\boldsymbol{x})$ are considered inputs to the event. If an event is meant to return data, it must do so by using of the belief base. To support failure recovery, we slightly modify how plans in the plan library $\Pi$ are converted into ConGolog procedures. Specifically, for each event $e(\boldsymbol{x})$, we define the following procedure (and make procedure achieve $_{e}(\boldsymbol{x})$ simply call achieve $_{e}^{\prime}(\boldsymbol{x},[1, \ldots, 1])$ :

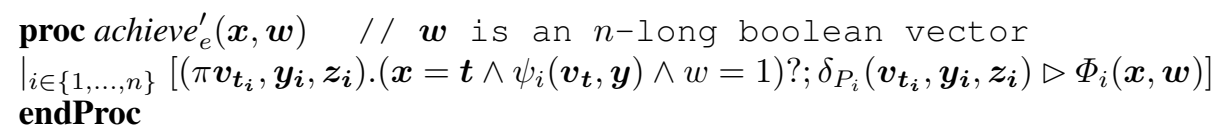

where $\Phi_{i}(\boldsymbol{x}, \boldsymbol{w}) \stackrel{\text { def }}{=} \operatorname{achieve}_{e}^{\prime}\left(\boldsymbol{x},\left[w_{1}, \ldots, w_{i-1}, 0, w_{i+1}, \ldots, w_{n}\right]\right)$. 
The boolean vector $\boldsymbol{w}$ has one component per plan rule in the library for the event in question; its $i$-th component $w_{i}$ states whether the $i$-th plan in $\Pi$ is available for selection. Condition $\left(\boldsymbol{x}=\boldsymbol{t} \wedge \psi_{i}\left(\boldsymbol{v}_{\boldsymbol{t}}, \boldsymbol{y}\right) \wedge w=1\right)$ checks whether event $e(\boldsymbol{x})$ is relevant, applicable, and available. Program $\Phi_{i}$ determines the recovery strategy, in this case, recursively calling the procedure to achieve the event, but removing the current plan from consideration (by setting its component in $\boldsymbol{w}$ to 0 ). Due to the semantics of $\triangleright$, recovery would only be triggered if procedure achieve $_{e}^{\prime}(\boldsymbol{x}, \boldsymbol{w})$ may execute one step, which implies that there is indeed an available plan that is relevant and applicable for the event.

It turns out that these are the only modifications to the encoding of Section 3 required to mimic the behavior of CAN agents with failure handling in the IndiGolog high-level language.

Theorem 4. Theorems 2 and 3 hold for CAN agents that drop impossible intentions and perform failure recovery under the extended translations into IndiGolog agents. ${ }^{7}$

More interestingly, the proposed translation can be adapted to accommodate several alternative accounts of execution and failure recovery. For example, goal failure recovery can be disallowed for an event by just taking $\Phi_{i}(\boldsymbol{x}, \boldsymbol{w}) \stackrel{\text { def }}{=} ?($ False $)$ above. Similarly, a framework under which any plan may be (re)tried for achieving a goal event, regardless of previous (failed) executions, is obtained by taking $\Phi_{i}(\boldsymbol{x}, \boldsymbol{w}) \stackrel{\text { def }}{=}$ achieve $_{e}(\boldsymbol{x})$. In this case, the event is "fully" re-posted within the intention.

The key point here is that, due to the fact that the BDI execution and recovery model is represented in our BDI IndiGolog at the object level, one can go even further and design more sophisticated accounts of execution and failure recovery for BDI agents. It is straightforward, for instance, to model the kind of goal failure recovery originally proposed for AgentSpeak, in which the system would automatically post a distinguished failure goal (denoted ! $-g$ ); the programmer may then choose to provide handling plan could, for example, carry out some clean-up tasks and even repost the failed event $[22,3]$. This type of behavior can be easily achieved by taking $\Phi_{i}(\boldsymbol{x}, \boldsymbol{w}) \stackrel{\text { def }}{=}$ ach $_{\text {fail_e }}(\boldsymbol{x}) ; ?($ False $)$, and allowing the programmer to provide plan rules in the library for handling the special event fail_e $e(\boldsymbol{x})$. Notice that the event is posted so it would eventually create a new intention all-together; the current plan would then immediately be blocked/failed.

\section{Discussion}

In this paper, we have shown how one can effectively program BDI-style agent systems in the situation calculus-based IndiGolog high-level programming language. The benefits of this are many. First, we gain a better understanding of the common features of BDI agent programming languages and "Golog-like" high-level programming languages, as well as of what is specific to each type of language, and what is required

\footnotetext{
${ }^{7}$ Note that for this to go through, we have to extend the translation $\delta_{P}$ (Section 3.2) of programs of the form $(\Delta)$ to set the bit vector $w$ in achieve $_{e}(\boldsymbol{x} . w)$ properly (i.e., to 1 iff the alternative is in the $(\Delta)$ ).
} 
to reproduce BDI languages in the latter. We also get a new classical-logic situation calculus-based semantics for BDI agent programming languages. This opens many avenues for enhancing the BDI programming paradigm with reasoning capabilities, for instance, model-based belief update capabilities, lookahead planning capabilities, plan/goal achievement monitoring capabilities, etc. In fact, the situation calculus and basic action theories provide a rich and and well-studied logical framework for specifying the belief update and planning part of agent programming languages.

It might be said that our bisimulation results are not very surprising, as both BDI languages and IndiGolog are universal programming languages. However, the simplicity and modularity of our encoding shows that the relationship between BDI languages and IndiGolog is actually fairly close. Representing a BDI-style plan library (including the association between a plan and the event/goal it addresses) in IndiGolog is quite straightforward: each plan becomes an alternative in an achievement procedure associated with the event/goal; event-directed plan invocation can be done by calling the event's achievement procedure. The key feature that is missing, BDI-style eventdirected plan triggering, can in fact be added by incorporating into the IndiGolog program an "event server" that calls the event's achievement procedure; such a server can easily be programmed using IndiGolog's concurrent iteration construct. We also show how the simple BDI language approach to belief update can be modeled in the situation calculus, using action theories with a simple type of successor state axiom and $a d d_{b_{i}}$ and $\operatorname{del}_{b_{i}}$ actions. This then gives us a nice framework for defining more sophisticated belief update approaches. As well, we have shown that failure recovery mechanisms can be added to IndiGolog by introducing constructs such as the "try" construct $\triangleright$, very much as has been done in some BDI-languages.

One could also argue that the approach we follow decreases the "separation of concerns" in that both the agent program and the generic BDI execution engine are encoded into a single IndiGolog high-level program, meaning that the agent program and the BDI interpreter are no longer separated. To avoid this, one could develop an alternative account where one gives a situation calculus semantics for the BDI execution cycle at the meta-level, by re-defining what a BDI-IndiGolog agent is and what counts as an "online" execution for such agents. In this paper, however, we intended to keep the IndiGolog framework as intact as possible. Also, by encoding the BDI engine in a logical/object language (as an IndiGolog program), one can formally express (and prove) properties of programs and of the programming language in the (situation calculus) object language. Nonetheless, the alternative approach is of interest and we are in fact working on it.

There has only been limited work on relating "Golog-like" and BDI programming languages. Hindriks et al. [16] show that ConGolog can be bisimulated by the agent language 3APL under some conditions, which include the agent having complete knowledge. In [14], it is also shown that AgentSpeak can be encoded into 3APL. Our results, thus, are complementary, in showing the inverse relationship.

Also related is the work of Gabaldon [12] on encoding Hierarchical Task Network (HTN) libraries in ConGolog. There are similarities between our work and his in the way procedural knowledge is encoded in ConGolog. This is is not surprising, as HTN planning systems and BDI agents have many similarities [11]. But note that in HTNs, and hence in Gabaldon's translation, the objective is planning and not reactive execu- 
tion. We on the other hand, focus on capturing the typical execution regime of BDI agent systems, rather than on performing lookahead planning to synthesize a solution. As a result, we address issues such as external events and plan failure that do not arise in HTN planning.

Finally, we note that in this work we have mostly focused on the core features of BDI systems, and have not dealt with more advanced features present in some more recent versions of BDI systems. For instance, the full CAN [29,27] language as well as 2APL [7] and Jason [3] support so-called declarative goals, goals which go beyond "events" by decoupling goal success/failure from plan success/failure. However, the way such advanced features have been added to different BDI languages is not always uniform. More work is needed to handle those advanced features in an encoding of BDI programming into IndiGolog.

Acknowledgments We thank the reviewers for their interesting comments. This work was supported by Agent Oriented Software and the Australian Research Council (grant LP0882234) and the National Science and Engineering Research Council of Canada.

\section{References}

1. R. A. Belecheanu, S. Munroe, M. Luck, T. Payne, T. Miller, P. McBurney, and M. Pechoucek. Commercial applications of agents: Lessons, experiences and challenges. In Proceedings of Autonomous Agents and Multi-Agent Systems (AAMAS), pages 1549-1555. ACM Press, Hakodate, Japan 2006.

2. S. S. Benfield, J. Hendrickson, and D. Galanti. Making a strong business case for multiagent technology. In Proceedings of Autonomous Agents and Multi-Agent Systems (AAMAS), pages 10-15, New York, NY, USA, 2006. ACM Press.

3. R. H. Bordini, J. F. Hübner, and M. Wooldridge. Programming Multi-agent Systems in AgentSpeak Using Jason. Wiley Series in Agent Technology. Wiley, 2007.

4. M. E. Bratman. Intentions, Plans, and Practical Reason. Harvard University Press, 1987.

5. P. Busetta, R. Rönnquist, A. Hodgson, and A. Lucas. JACK intelligent agents: Components for intelligent agents in Java. AgentLink Newsletter, 2, Jan. 1999. Agent Oriented Software Pty. Ltd.

6. P. R. Cohen and H. J. Levesque. Intention is choice with commitment. Artificial Intelligence Journal, 42:213-261, 1990.

7. M. Dastani. 2APL: a practical agent programming language. Autonomous Agents and MultiAgent Systems, 16(3):214-248, June 2008.

8. G. De Giacomo, Y. Lespérance, and H. J. Levesque. ConGolog, a concurrent programming language based on the situation calculus. Artificial Intelligence Journal, 121(1-2):109-169, 2000.

9. G. De Giacomo, Y. Lespérance, H. J. Levesque, and S. Sardina. IndiGolog: A high-level programming language for embedded reasoning agents. In R. H. Bordini, M. Dastani, J. Dix, and A. E. Fallah-Seghrouchni, editors, Multi-Agent Programming: Languages, Platforms and Applications, chapter 2, pages 31-72. Springer, 2009.

10. D. Dennett. The Intentional Stance. The MIT Press, 1987.

11. J. Dix, H. Muñoz-Avila, D. S. Nau, and L. Zhang. IMPACTing SHOP: Putting an AI planner into a multi-agent environment. Annals of Mathematics and Artificial Intelligence, 37(4):381-407, 2003. 
12. A. Gabaldon. Programming hierarchical task networks in the situation calculus. In Proc. of AIPS'02 Workshop on On-line Planning and Scheduling, Toulouse, France, April 2002.

13. M. P. Georgeff and F. F. Ingrand. Decision making in an embedded reasoning system. In Proceedings of the International Joint Conference on Artificial Intelligence (IJCAI), pages 972-978, Detroit, USA, 1989.

14. K. V. Hindriks, F. S. de Boer, W. van der Hoek, and J.-J. Meyer. A formal semantics for an abstract agent programming language. In Proceedings of the International Workshop on Agent Theories, Architectures, and Languages (ATAL), pages 215-229, 1998.

15. K. V. Hindriks, F. S. de Boer, W. van der Hoek, and J.-J. Meyer. Agent programming in 3APL. Autonomous Agents and Multi-Agent Systems, 2:357-401, 1999.

16. K. V. Hindriks, Y. Lespérance, and H. J. Levesque. An embedding of ConGolog in 3APL. In Proceedings of the European Conference in Artificial Intelligence (ECAI), pages 558-562, 2000.

17. H. J. Levesque, R. Reiter, Y. Lespérance, F. Lin, and R. B. Scherl. GOLOG: A logic programming language for dynamic domains. Journal of Logic Programming, 31:59-84, 1997.

18. M. Ljungberg and A. Lucas. The OASIS air-traffic management system. In Proceedings of the Pacific Rim International Conference on Artificial Intelligence (PRICAI), Seoul, Korea, 1992.

19. J. McCarthy and P. J. Hayes. Some philosophical problems from the standpoint of artificial intelligence. Machine Intelligence, 4:463-502, 1969.

20. A. Pokahr, L. Braubach, and W. Lamersdorf. JADEX: Implementing a BDI-infrastructure for JADE agents. EXP - in search of innovation (Special Issue on JADE), 3(3):76-85, 9 2003.

21. M. E. Pollack. The uses of plans. Artificial Intelligence Journal, 57(1):43-68, 1992.

22. A. S. Rao. Agentspeak(L): BDI agents speak out in a logical computable language. In W. V. Velde and J. W. Perram, editors, Proceedings of the Seventh European Workshop on Modelling Autonomous Agents in a Multi-Agent World. (Agents Breaking Away), volume 1038 of Lecture Notes in Computer Science (LNCS), pages 42-55. Springer-Verlag, 1996.

23. A. S. Rao and M. P. Georgeff. Modeling rational agents within a BDI-architecture. In Proceedings of Principles of Knowledge Representation and Reasoning (KR), pages 473484, 1991.

24. A. S. Rao and M. P. Georgeff. An abstract architecture for rational agents. In Proceedings of Principles of Knowledge Representation and Reasoning (KR), pages 438-449, San Mateo, CA, 1992.

25. R. Reiter. Knowledge in Action. Logical Foundations for Specifying and Implementing Dynamical Systems. The MIT Press, 2001.

26. S. Sardina, G. De Giacomo, Y. Lespérance, and H. J. Levesque. On the semantics of deliberation in IndiGolog - From theory to implementation. Annals of Mathematics and Artificial Intelligence, 41(2-4):259-299, Aug. 2004.

27. S. Sardina and L. Padgham. Goals in the context of BDI plan failure and planning. In E. H. Durfee, M. Yokoo, M. N. Huhns, and O. Shehory, editors, Proceedings of Autonomous Agents and Multi-Agent Systems (AAMAS), pages 16-23, Hawaii, USA, May 2007. ACM Press.

28. M. Thielscher. FLUX: A logic programming method for reasoning agents. Theory and Practice of Logic Programming, 5(4-5):533-565, 2005. Special Issue of Theory and Practice of Logic Programming on Constraint Handling Rules.

29. M. Winikoff, L. Padgham, J. Harland, and J. Thangarajah. Declarative \& procedural goals in intelligent agent systems. In Proceedings of Principles of Knowledge Representation and Reasoning (KR), pages 470-481, Toulouse, France, April 2002. 